\title{
Transient sources at the highest angular resolution
}

\author{
Maria Massi* \\ Max-Planck-Institut für Radioastronomie, Bonn, Germany \\ E-mail: mmassi@mpifr-bonn.mpg.de
}

By definition transients are sudden events, some, like supernovae, are catastrophic, while others might be due to recurrent phenomena. The aim of studying transients is to reveal the physical conditions causing them, in this sense ideal targets for monitoring are transients in binary systems. In these systems the physical process responsible for the transient depends directly or indirectly on the interaction of the two components of the system. Here I report on transients in stellar binary systems at two extremes of stellar evolution: a T Tauri system formed by two young low mass stellar objects, and X-ray binary systems formed by a star and a neutron star or a black hole, i.e., end points in the life of massive stars. VLBI observations of the young binary system V773 Tau A resolve the binary separation and can be overlapped with the optical frame. Consecutive VLBI observations showing the evolution of the radio emission with respect to the two stellar objects are an unvaluable tool for a better understanding of the magnetic field topology of T Tauri stars. The characteristics of radio jets in X-ray binaries are summarised and compared with those of the radio emission of the gamma-ray binary LS I $+61^{\circ} 303$. Timing analysis of radio and Fermi-LAT observations provide constraints for theoretical models that can be tested by VLBI observations.

12th European VLBI Network Symposium and Users Meeting,

7-10 October 2014

Cagliari, Italy

${ }^{*}$ Speaker. 


\section{Introduction}

Radio transients [1] are observed from the nearby Sun to cosmological distances. A classification of transients following the physical process in act is complicated: different physical processes, as magnetic reconnection, shocks, electron beams, etc., may work in the same object. A possible classification is that based on the kind of astronomical objects where the transient occur: cool stars, supernovae, pulsars and jets. Straightforward and based in differences in timescale is the classification of radio transients in fast transients and slow transients. Fast radio transients may have timescales of nanoseconds to minutes and they typically are discovered in time-series data. The slow radio transients, variable on timescales of seconds up to years, are typically discovered in images and are those described here. Transients in binary systems, where the interaction between the components is the cause of the transient, are ideal targets for monitoring studies. In the next section I will therefore examine slow radio transients in binary systems.

\section{Transients in a young-stellar system}

The binary system V773 Tau A [2] shows outstanding magnetic activity demonstrated by radio and X-ray flares and the presence of large, cool, photospheric spots. A periodicity of $51.1 \mathrm{~d}$, the same as the orbital one, has been discovered in the radio flaring activity via long-term monitoring with the Effelsberg 100-m telescope: large flares cluster around periastron passage [3]. The orbit, with an eccentricity of $e=0.27$ [2], results at apastron in a distance of 52 stellar radii $\left(1 R_{*}=2\right.$ solar radii) between the two stars, and at periastron of 30 stellar radii. If the strong flaring activity at periastron is due to interacting coronae, then the magnetic structures should have sizes of at least 15 stellar radii. Observations at $90 \mathrm{GHz}$ around periastron have monitored the onset and decay of a large flare and show that the decay of few hours is consistent with continuous leakage of the emitting relativistic electrons from a magnetic structure of 10-20 stellar radii [4]. There exists a close relationship between flares and interaction of magnetic structures. In fact, as observed on the Sun, flares can be triggered by interactions between new and older emergences of magnetic flux in the same area [5]. Part of the magnetic energy released during reconnection goes to accelerate a fraction of the thermal electrons trapped in the flaring coronal loop and a population of relativistic electrons is produced [6,7]. These relativistic particles gyrating around the magnetic field lines of

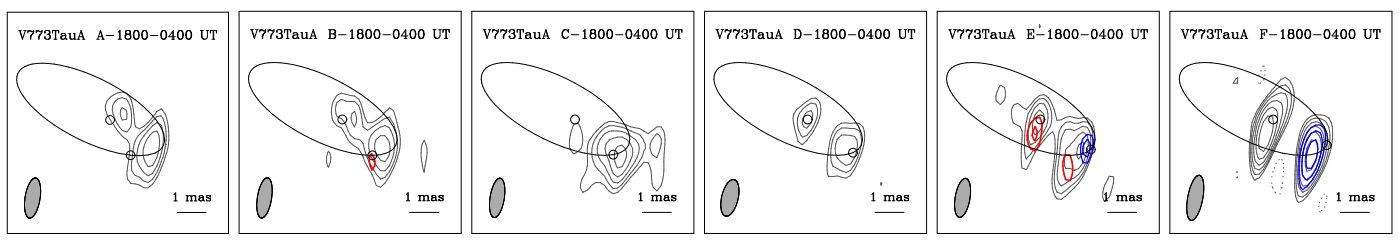

Figure 1: Phase-referenced images of consecutive (25 February - 2 March 2010) 8.4 GHz VLBA+EB observations of V773 Tau A. Overlaid is the orbit of the binary stellar system; the circle of $4 R_{*}$ centered at each star's position indicates a symmetrical corona of an average size of $3 R_{*}$ (the stellar radius corresponds to 0.07 mas at $132.8 \mathrm{pc}$ distance). The restoring beam is shown in the bottom left corner of each map; it is on average of $(0.5 \times 1.4)$ mas. Circularly polarized emission (traced in colour) is associated with the secondary star in $\mathrm{E}$ and $\mathrm{F}$ with $\mathrm{V} / \mathrm{I}$ equal to $-4.3 \% \pm 0.4 \%,-8.0 \% \pm 0.3 \%$ and also to the primary in $\mathrm{E}$, with opposite polarization sense and V/I of $4.0 \% \pm 0.4 \%$ [8]. 
the loop, where they are confined, generate synchrotron emission in the radio band. Applying this knowledge of solar flares to V773 Tau A, it is clear that in this system the observed relationship between intensity of the flare occurrence and the distance between the two stars indicates another, new mechanism of magnetic interaction, that of interacting coronae. In this case magnetic reconnection would take place far out from the stellar surfaces, where the two coronae interact with each other. Figure 1 shows phase referenced maps of consecutive VLBA+EB observations aimed to spatially trace the flare evolution and polarized emission around periastron passage where the intensity of the flares is highest [8]. The visual orbit is derived from an interferometric-spectroscopic orbital model [2] and the primary star is overlapped with the North-East feature in the map of run D. By using the images, the total flux density monitoring, model fitting and the information from circular polarization (here in colour) we have a powerful tool for a better understanding of the physical process triggering the transient and of the magnetic field topology of weak-lined T Tauri stars.

\section{Radio transients in $\mathrm{X}$-ray binaries}

$\mathrm{X}$-ray binaries are stellar systems formed by a compact object (black hole or neutron star) and a normal star. Neutron stars in X-ray binaries may have quite different values of magnetic field, being the range of $\mathrm{B}$ about $10^{8}-10^{12} \mathrm{G}$; however, X-ray binaries with a radio emitting jet, systems called "microquasars", have as compact object either an accreting black hole or an accreting neutron star with low magnetic field $\left(10^{8} \mathrm{G}\right)$ [9]. Radio jets have been imaged at high resolution for the three neutron star systems: Scorpius X-1 [10], Circinus X-1 [11] and Cygnus X-2 [12]. The statistically more powerful radio jets associated to accreting black holes have quite well studied spectral characteristics. There are two kind of jets; the steady jet and the transient jet [13]. The origin of a steady jet is relatively well understood as a result of magneto-rotational processes: an initial vertical magnetic field treading the accretion disk is bent by the differentially rotating disk, then magnetic pressure gradient accelerates plasma out of system and magnetic tension pinches and collimates the outflow into a jet [14]. Changes in the plasma density and in the strength of the magnetic field along the conical jet create distinct regions of synchrotron emission with each region contributing with a spectrum peaking at a different frequency. Then the overall spectrum, observed with a spatial resolution insufficient to resolve the individual parts of the jet, will be almost flat, i.e., with a spectral index $\alpha \sim 0$, or inverted, i.e., $\alpha>0$ [13, 15] (flux density $S \propto v^{\alpha}$ ). The other type of jet associated to microquasars is the so called transient jet. It corresponds to an optically
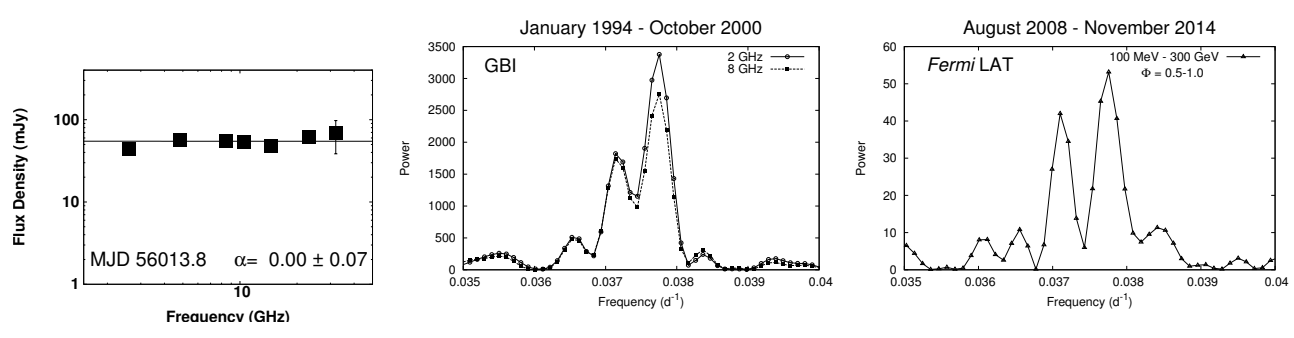

Figure 2: Left: Flat radio spectrum of LS I $+61^{\circ} 303$ measured with the Effelsberg 100-m telescope at 2.8, $4.85,8.35,10.45,14.3,23$ and $32 \mathrm{GHz}$ [24]. Center: Periodogram of GBI radio data. Right: Periodogram of Fermi-LAT data (see Sect. 3.1). 

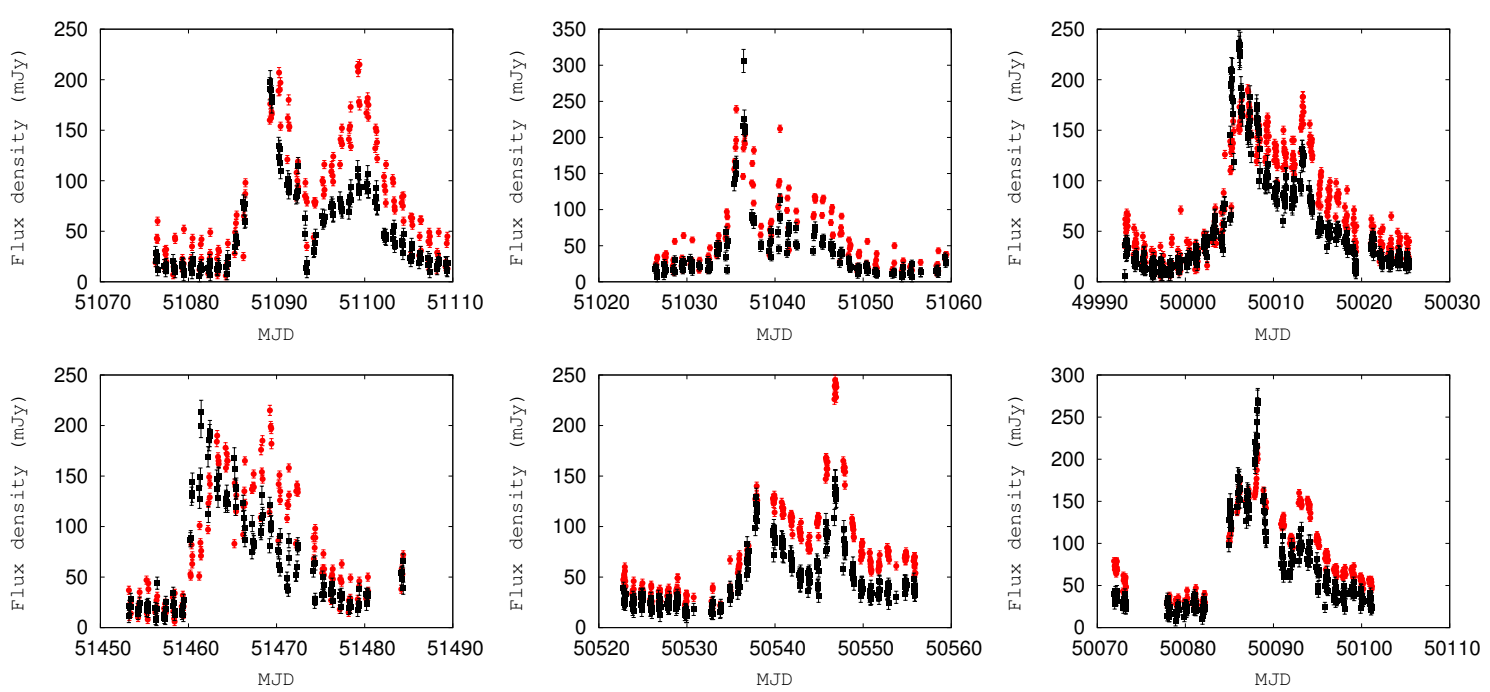

Figure 3: Light radio curves of $\mathrm{LS} \mathrm{I}+61^{\circ} 303$ at $2 \mathrm{GHz}$ (red) and $8 \mathrm{GHz}$ (black) GBI data vs time (MJD) [25]. An optically thin outburst follows an optically thick outburst as in XTE J1752-223 (see Sect. 3).

thin radio outburst (i.e., $\alpha<0$ ) and occurs always after the flat spectrum phase. The transient jet is thought to be associated to shocks where high relativistic plasma catches up with the preexisting slower-moving material of the steady jet [13]. The switch from the self-absorbed jet to the transient one presents differences in the different sources. In the microquasar GRS 1915+105 the switch is between a steady, plateau state and the optically thin outburst [Fig. 1, in 16], whereas in XTE J1752-223 the switch is between an optically thick outburst and an optically thin outburst [Fig. 1, in 17]. A unique case of a radio outburst from an X-ray binary having as compact object a non-accreting neutron star is PSR B1259-63. The neutron star is in this case a young pulsar, i.e., having both a strong $B\left(10^{12} \mathrm{G}\right)$ and a fast rotation (msec period). Around periastron passage the interaction of the pulsar wind and the wind of the companion star generates an optically thin outburst [18] mapped at high resolution [19].

\subsection{Latest results on a transient source: The gamma-ray binary $\mathrm{LS}$ I $+61^{\circ} 303$}

The stellar system LS I $+61^{\circ} 303$ is formed by a compact object and a Be star in an eccentric orbit, $e=0.72 \pm 0.15$ [20]. High resolution radio images show a structure that not only changes position angle, but it is even sometimes one-sided and at other times two-sided. This suggested the hypothesis of LS I $+61^{\circ} 303$ being a precessing microquasar [21]. A precession of the jet leads to a variation in the angle between the jet and the line of sight, and therefore to variable Doppler boosting. The result is both a continuous variation in the position angle of the radio-emitting structure and its flux density. The rapid variations were alternatively interpreted [22] to be due to a young pulsar whose wind enters in collision with the disc/wind of the companion star, i.e., a system as PSR B1259-63. As we saw in the previous section, the radio spectral index for a radio emitting jet of a microquasar and that of a radio emitting nebula associated to interacting winds are quite different. A flat/inverted spectrum is expected for a radio jet, followed by an optically thin outburst in case of a transient. Just an optically thin outburst is expected for the pulsar nebula. It is therefore worth to examine the radio characteristics of $\mathrm{LS} \mathrm{I}+61^{\circ} 303$ in detail. 

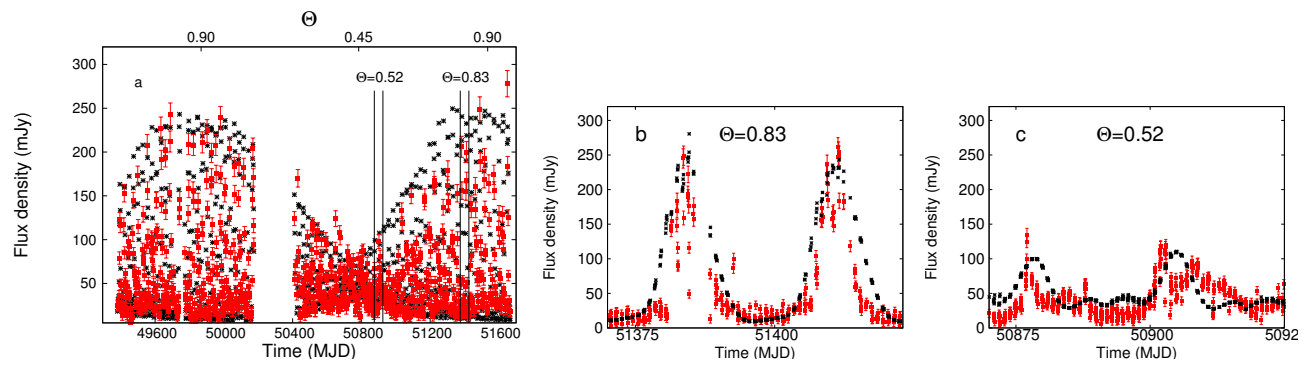

Figure 4: Long-term modulation in LSI+61303. a) $8 \mathrm{GHz}$ GBI observations (red) and model (black) data [32]. $b$ and c) Zoom of two intervals of Fig. 4 a.

VLA observations [23] measured a flat spectrum extending from $1.5 \mathrm{GHz}$ to $22 \mathrm{GHz}$. Effelsberg 100-m telescope observations (Fig. 2), reveal a flat spectrum up to $32 \mathrm{GHz}$ [24]. Observations at two frequencies, $2 \mathrm{GHz}$ and $8 \mathrm{GHz}$ of the Green Bank Interferometer (GBI) are given in Fig. 3. As in Fig. 4 of [25], it is also evident in Fig. 3 that in LS I $+61^{\circ} 303$ two consecutive outbursts occur: there is a first outburst either dominating at $8 \mathrm{GHz}$ (i.e., with inverted spectrum, $\alpha>0$ ) or with flat spectrum (comparable flux at the two frequencies) and then there follows another outburst, this one clearly dominating at $2 \mathrm{Ghz}$ (i.e., $\alpha<0$ ). The system LS I $+61^{\circ} 303$ does not only show the self-absorbed jet but in addition there it follows a transient jet, as typical for microquasars. Finally, following the hypothesis that a short X-ray burst observed in the direction of LS I $+61^{\circ} 303$ could be attributed to this system and not to the other X-ray source that is located in the same field of view, possible implications for having a magnetar in a binary system have been analysed [26]. The presence of another X-ray candidate on the one hand and the radio observations well consistent with those of microquasars on the other hand do corroborate the hypothesis of LS I $+61^{\circ} 303$ to be a precessing microquasar.

Deriving the precessional period from radio images is not straightforward, because the radio structures reflect the variation of the projected angle on the sky plane and therefore a combination of the ejection angle, $\eta$, and inclination. The most powerful tool to determine the precession is the radio astrometry: following the shift of the peak of successive high resolution radio images we determined a period of $27-28 \mathrm{~d}$ for the precession [27]. An independent estimate of the precession comes from the timing analysis. In fact, precession implies a periodical Doppler boosting, that is periodical changes in the flux density and this can be revealed by timing analysis. A timing analysis of the 6.7 years of GBI radio data of LS I $+61^{\circ} 303$ has revealed indeed (Fig. 2) two rather close frequencies: $P_{1}=\frac{1}{v_{1}}=26.49 \pm 0.07$ days and $P_{2}=\frac{1}{v_{2}}=26.92 \pm 0.07$ days [28]. The period $P_{1}$ agrees with the value of $26.4960 \pm 0.0028$ days [29] associated to the orbital period of the binary system and corresponds to the predicted periodical accretion peak along an eccentric orbit [30]. The period $P_{2}$ agrees well with the estimate by radio astrometry of $27-28$ days for the precession period. Recently [31], Lomb-Scargle analysis of Fermi-LAT data around apoastron revealed (Fig. 2) the same periodicities $P_{1_{\gamma}}=26.48 \pm 0.08 \mathrm{~d}, P_{2_{\gamma}}=26.99 \pm 0.08 \mathrm{~d}$. The similar behaviour of the emission at high $(\mathrm{GeV})$ and low (radio) energy towards apoastron is a hint for these emissions to be caused by the same population of electrons in a precessing jet.

The beating of the two periodicities, $P_{1}$ and $P_{2}$, likely gives rise to the so called "long-term" modulation present in LS I $+61^{\circ} 303$. The peak flux density of the periodical radio outburst ex- 
hibits in fact a modulation of $1667 \pm 8 \mathrm{~d}$ [29]. The beating of the two frequencies determined in the GBI timing analysis gives straightforward the long-term modulation as $\frac{1}{v_{1}-v_{2}}=1667$ days [28]. Indeed, a physical model [32] for LS I $+61^{\circ} 303$ of synchrotron emission from a precessing $\left(P_{2}\right)$ jet, periodically $\left(P_{1}\right)$ refilled with relativistic particles, produces a maximum (shown here in Fig. $4 \mathrm{a}, \mathrm{b}$ ) when the jet electron density is at its maximum and the approaching jet forms the smallest possible angle with the line of sight. This coincidence of the highest number of emitting particles and the strongest Doppler boosting of their emission occurs with the frequency of $v_{1}-v_{2}$ creating the long-term modulation observed in LS I $+61^{\circ} 303$. As one can see the model reproduces in fact also the minimum of the log-term modulation (Fig. 4 c) corresponding at ejections when the approaching jet forms the largest possible angle with the line of sight [32].

The model can determine the variation of the ejection angle, $\eta$, at the epochs of VLBI observations. It is therefore of interest to compare predicted variations of $\eta$ with the observed variations in position angle of the radio structures in high resolution images. The trend of $\eta$ vs $\Phi\left(P_{1}\right)$ (orbital phase) is shown in Fig. 5 together with VLBA images [27,32]. The plot of $\eta$ vs $\Phi$ reveals that run $\mathrm{H}$ was performed at the minimum $\eta$ and $\mathrm{D}$ was performed nearly at the maximum $\eta$. These two extreme situations of $\eta$ for the two runs, $\mathrm{H}$ and $\mathrm{D}$, are sketched in Fig. 5 (right corner). This important information implies that two runs at similar $\eta$, but one performed before and the other after run $\mathrm{D}$, refer to two jets pointing to opposite directions with respect to the axis of the precession cone (Fig. 5, left corner). If the model is correct, the position angle of the associated radio structures should reflect this different orientation. Indeed, the structure for run E points towards South-West, whereas runs $\mathrm{C}$ and $\mathrm{J}$ show structures pointing to South-East. Similarly, the structure at run B points towards East whereas that for run F points towards West. Finally, for A, I, H, G our model results in a low $\eta$ angle; this would correspond to a jet pointing closest to the earth (i.e., a micro-blazar). One can see that the related VLBA structures develop indeed a North-South feature.

To conclude this short review, there are different mechanisms producing transients in binary systems. Intrabinary interaction of the magnetic structures of the two stellar objects in V773 Tau A is likely the origin of the transient in this system. Variable accretion in an eccentric orbit and consequent refilling of a precessing jet is likely the cause for the modulated transient in the gammaray binary $\mathrm{LS} \mathrm{I}+61^{\circ} 303$.

\section{Acknowledgments}

We thank Eduardo Ros and Frederic Jaron for careful reading of the manuscript and helpful comments and suggestions. This research is based on observations with the 100-m telescope of the MPIfR (Max-Planck-Institut für Radioastronomie) at Effelsberg. The Very Long Baseline Array is operated by the National Radio Astronomy Observatory, a facility of the National Science Foundation operated under cooperative agreement by Associated Universities, Inc. This work has made use of public Fermi-LAT data obtained from the High Energy Astrophysics Science Archive Research Center (HEASARC), provided by NASA Goddard Space Flight Center.

\section{References}

[1] J. M. Cordes, T. J. W. Lazio, M. A. McLaughlin, The dynamic radio sky, New Astronomy Reviews 48 (1459) 2004. 


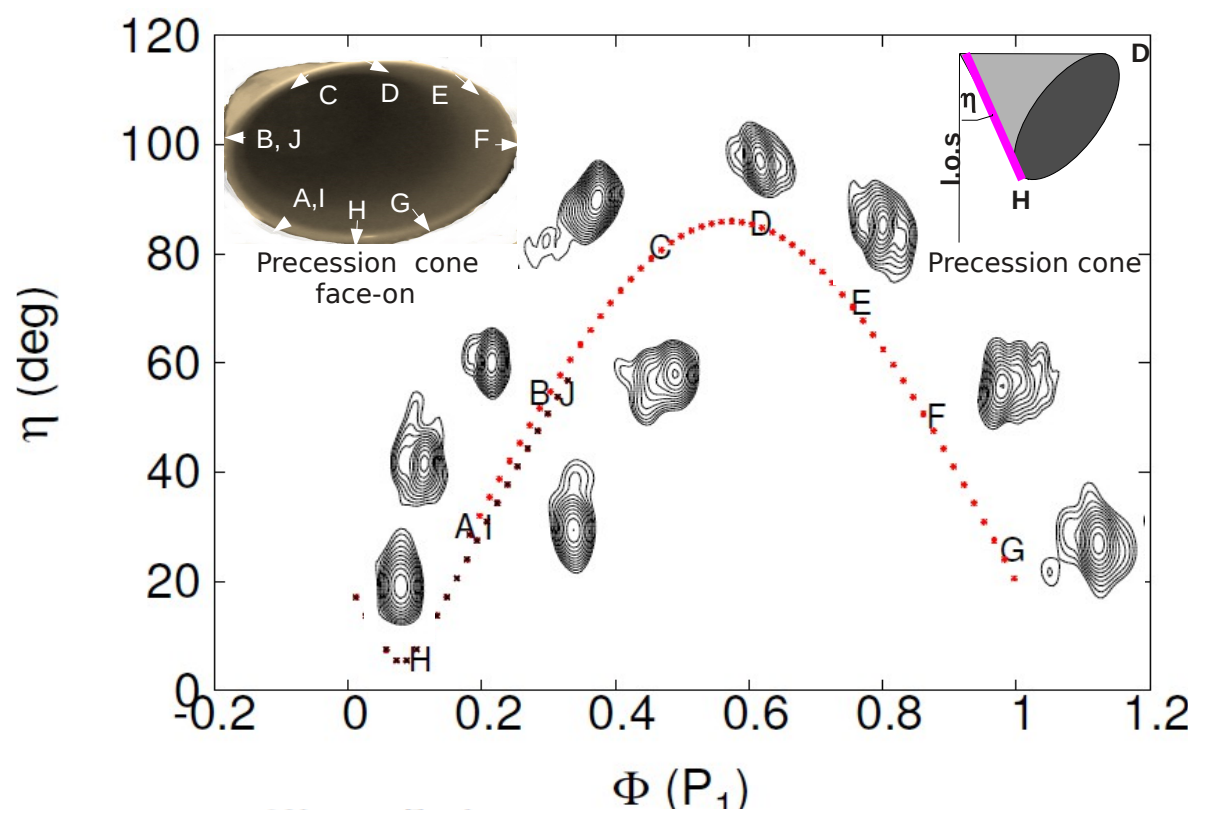

Figure 5: VLBA images and related variations of the ejection angle $\eta$ because of precession (see Sect. 3.1) [32]

[2] A. F. Boden, G. Torres, A. I. Sargent, et al., Dynamical Masses for Pre-Main-Sequence Stars: A Preliminary Physical Orbit for V773 Tau A, ApJ 670 (1214) 2007.

[3] M. Massi, K. Menten, J. Neidhöfer, Periodic radio flaring on the T Tauri star V 773 Tauri, A\&A, 382 (152) 2002.

[4] M. Massi, J. Forbrich, K. M. Menten, et al., Synchrotron emission from the T Tauri binary system V773 Tauri A, A\&A 4539592006.

[5] M. Nishio, K. Yaji, T. Kosugi, H. Nakajima, T. Sakurai, Magnetic Field Configuration in Impulsive Solar Flares Inferred from Coaligned Microwave/X-Ray Images, ApJ 489 (976) 1997.

[6] Drake, M.A. Shay, W. Thongthai, M. Swisdak, Production of Energetic Electrons during Magnetic Reconnection, Physical Review Letters 94 (95001) 2005.

[7] M. Massi, E. Ros, K. M. Menten, et al., Interacting coronae of two T Tauri stars: first observational evidence for solar-like helmet streamers, A\&A 4804892008.

[8] M. Massi, E. Ros, D. Boboltz, et al., Interacting coronae of two T Tauri stars, MemSAI 843592013.

[9] M. Massi, M. Kaufman Bernado', Magnetic field upper limits for jet formation, A\&A 47712008.

[10] E. B. Fomalont, B. J. Geldzahler, C. F. Bradshaw, Scorpius X-1: The Evolution and Nature of the Twin Compact Radio Lobes Ap. J. $\mathbf{5 5 8} 2832001$.

[11] V. Tudose, R.P. Fender, C.R. Kaiser, et al., The large-scale jet-powered radio nebula of Circinus X-1, MNRAS 3724172006.

[12] R. E. Spencer, A. P. Rushton, M. Balucinska-Church, et al., Radio and X-ray observations of jet ejection in Cygnus X-2, MNRAS 435 L48 2013. 
[13] R. P. Fender, T. M. Belloni, E. Gallo. Towards a unified model for black hole X-ray binary jets MNRAS 35511052004.

[14] D. Meier, S. Koide, Y. Uchida, Magnetohydrodynamic Production of Relativistic Jets, Science 29184 2001.

[15] C. Kaiser, The flat synchrotron spectra of partially self-absorbed jets revisited, MNRAS 3671083 2006.

[16] V. Dhawan, I. F. Mirabel, L. F. Rodriguez, AU-scale synchrotron jets and superluminal ejecta in GRS 1915+105, Ap. J. $\mathbf{5 4 3} 3732000$.

[17] C. Brocksopp, S. Corbel, A. Tzioumis, et al., XTE J1752-223 in outburst: a persistent radio jet, dramatic flaring, multiple ejections and linear polarization, MNRAS 4329312013.

[18] T. W. Connors, S. Johnston, R. N. Manchester, D. McConnell, The 2000 periastron passage of PSR B1259-63, MNRAS 33612012002.

[19] J. Moldón, S. Johnston, M. Ribó, J. M. Paredes, A. T. Deller, Discovery of extended and variable radio structure from the gamma-ray binary PSR B1259-63/LS 2883, Ap. J. 732 L10 2011.

[20] J. Casares, I. Ribas, J.M. Paredes, J. Martí, C. Allende Prieto Orbital Parameters of the Microquasar $L S I+61303$, MNRAS 36011052005.

[21] M. Massi, M. Ribó, J. M. Paredes, et al., Hints for a fast precessing relativistic radio jet in LS I +61303, A\&A 414 L1 2004.

[22] V. Dhawan, A. Mioduszewski, M. Rupen, LS I +61 303 is a Be-Pulsar binary, not a Microquasar, POS (MQW6) 0522006.

[23] P. C. Gregory, A. R. Taylor, D. Crampton, et al., The radio, optical, X-ray, gamma-ray star LSI +61 deg 303, Astron. J. 8410301979.

[24] L. Zimmermann, L. Fuhrmann, M. Massi, The flat radio spectrum of LS I+61 303 and its evolution during outburst, A\&A 2015 submitted.

[25] M. Massi, M. Kaufman Bernado', Radio Spectral Index Analysis and Classes of Ejection in $L S I+61303, A \& A \mathbf{7 0 2} 12009$.

[26] D. F. Torres, N. Rea, P. Esposito, J. Li, Y. Chen, S. Zhang, A Magnetar-like event from LS I +61303 and its nature as a gamma-ray binary, Ap. J. 7441062012.

[27] M. Massi, E. Ros, L. Zimmermann, VLBA images of the precessing jet of LS I+61303, A\&A 540142 2012.

[28] M. Massi, F. Jaron, Long-term periodicity in LS I +61303 as beat frequency between orbital and precessional rate, A\&A 5541052013.

[29] P. Gregory, Bayesian Analysis of Radio Observations of the Be X-Ray Binary LS I +61303, Ap. J. 575 4272002.

[30] V. Bosch-Ramon, J. M. Paredes, G. E. Romero, M. Ribó, The radio to TeV orbital variability of the microquasar LS I +61303, A\&A 459 L25 2006.

[31] F. Jaron, M. Massi Discovery of a periodical apoastron GeV peak in LS I +61303, A\&A 5721052014.

[32] M. Massi, G Torricelli-Ciamponi, Intrinsic physical properties and Doppler boosting effects in LS I +61303, A\&A 564232014 . 